\title{
Route and Speed Optimization for Autonomous Trucks
}

\author{
Moncef Ilies Nasri ${ }^{\mathrm{a}, 1}$, Tolga Bektaşa ${ }^{\mathrm{a}, 2}$, Gilbert Laporte ${ }^{\mathrm{b}, 3}$ \\ ${ }^{a}$ CORMSIS and Southampton Business School, University of Southampton, Southampton SO17 1BJ, United \\ Kingdom \\ ${ }^{b}$ Canada Research Chair in Distribution Management and CIRRELT, HEC Montréal 3000, chemin de la \\ Côte-Sainte-Catherine, Montréal, Canada H3T 2A7
}

\begin{abstract}
Autonomous vehicles, and in particular autonomous trucks (ATs), are an emerging technology that is becoming a reality in the transportation sector. This paper addresses the problem of optimizing the routes and the speeds of ATs making deliveries under uncertain traffic conditions. The aim is to reduce the cost of emissions, fuel consumption and travel times. The traffic conditions are represented by a discrete set of scenarios, using which the problem is modeled in the form of two-stage stochastic programming formulations using two different recourse strategies. The strategies differ in the amount of information available during the decision making process. Computational results show the added value of stochastic modeling over a deterministic approach and the quantified benefits of optimizing speed.
\end{abstract}

Keywords: Autonomous Trucks, Stochastic programming, Speed optimization, Green VRP

\section{Introduction}

Autonomous trucks (ATs) are an emerging technology with potential to revolutionize the transportation sector. In Europe, a convoy of more than 12 ATs completed a European cross-border journey in April 2016 (The Guardian, 2016). The ATs formed platoons of two or three connected

5 trucks that drove close to each other, using the same speed, acceleration and steering profile. This was achieved by the use of an existing technology of adaptive cruise control where the trucks were connected via wireless technologies with a driver sitting in the lead truck. In such a setting, fuel savings of around $5 \%$ can be achieved by the lead truck, and between $10-15 \%$ for the follower trucks

\footnotetext{
${ }^{1}$ M.I.Nasri@soton.ac.uk

${ }^{2}$ T.Bektas@soton.ac.uk

${ }^{3}$ Gilbert.Laporte@cirrelt.ca
} 
(Tsugawa, 2012). In the US, the first freight shipment with an AT was made on the 25th of October 2016 (Wired.com, 2016). The truck was able to drive without driver assistance on the highway. It was able to assess traffic conditions, choose acceleration and speed, and had the ability to self steer to stay in the driving lane. The truck, however, needed a driver to control it in complex driving areas, such as cities.

The emergence of ATs can also address the problem of truck drivers shortage. In the US, there was a shortage of 48,000 truck drivers in 2015, which is predicted to be 175,000 by 2024 under the current trend (Costello and Suarez, 2015). ATs can help increase the overall traffic safety. Traffic crashes including trucks represent $10 \%$ of all crashes in the US, and more than $90 \%$ of accident causes are due to driver errors (Singh, 2015).

The introduction of ATs is likely to significantly improve transport efficiency and reduce pollution, one reason for which is the ability to better control vehicle speed, but subject to traffic conditions. To capture these two aspects, we consider in this paper a variant of the Vehicle Routing Problem (VRP), where the vehicles are assumed to be ATs. In addition to determining routes, we also compute optimal speeds at which ATs should travel on each leg of a route in order to minimize a comprehensive cost function that includes fuel costs. However, selecting the optimal travel speed depends on the traffic conditions in the network. In practice, vehicle speed is often affected by factors that are not known with certainty in advance, such as weather-related events or traffic congestion. Such events are likely to reduce the speed of vehicles and cause deterioration in the general traffic speed. If this is the case, vehicles cannot travel faster than the traffic speed. Ignoring this uncertainty at the planning stage may render optimal solutions computed using deterministic parameters sub-optimal or even infeasible, and in turn may result in a cost increase, depending on the traffic speed in practice. In addition, customers may experience large and unpredictable delays. Consequently, it makes sense to incorporate uncertainty in the optimization process to ensure that the planned solutions are robust against speed variations.

There have been several strategies proposed for the implementation of ATs, including lane reservation (Fang et al. 2013) and exclusive assignment of an existing infrastructure (Wu et al., 2017), although both are deemed to be expensive and restrictive with respect to the applicability of this new technology (Vanholme et al. 2013). A preferred feasible strategy, at least for the foreseeable future, is for ATs to share the same infrastructure with conventional vehicles (driven by humans) Vanholme et al. 2013). However, the latter option would introduce uncertain traffic conditions for 
ATs, which is precisely what we assume in this paper.

The problem we consider is similar to the Pollution-Routing Problem (PRP) introduced by Bektaş and Laporte (2011), in that we treat the choice of speed at which the vehicle will want to travel at on a given segment of road as a decision variable as part of devising the operational routing plans. This flexibility breaks away from the common assumption in traffic flow theory that drivers simply drive at maximum speed possible, and is used primarily for reducing fuel consumption. However, once en route, we do not assume that the vehicle is necessarily able to travel at the chosen speed on a given arc, as it may be constrained by uncertain traffic conditions that are not necessarily known at the time of planning. If this is the case, then the vehicle will have to travel at a speed lower than the one that was chosen, which impacts on fuel consumption and emissions. Our models take such situations into account in an explicit way. In this paper we model uncertainty as stochastic input parameters. We describe two stochastic programming models that differ with respect to the time at which the routing decisions are made. We consider a finite number of scenarios, each with a different traffic speed for each arc of the network. The first formulation is a two-stage stochastic programming model with complete recourse. In this case, the recourse variables correspond to the delays experienced in servicing the customers, and to the speed reduction needed when the planned speed is higher than the realized speed. The second formulation is also a two-stage stochastic programming model in which travel speeds are computed for every scenario separately, assuming that the realizations of the scenarios are revealed before the start of routing, in the expectation that further cost minimization will be achieved.

The three key contributions of this paper are as follows. (1) We describe two recourse strategies to mitigate the adverse effects of speed uncertainty on fuel consumption and greenhouse gases (GHG), where speed reductions are treated as an integral part of the recourse decisions for the first time, (2) using a stochastic programming methodology, we describe several two-stage stochastic programming formulations with recourse, and (3) we present results that numerically compare the recourse strategies and we offer insights into the relative performance of each strategy and discuss implications of the strategies on fuel consumption and total cost.

The remainder of this paper is structured as follows. Section 2 presents a brief review of the literature where routing and speed decisions were considered simultaneously. Section 3 introduces some technical background information. Mathematical models are described in Section 4. Section 5 presents computational experiments, and conclusions follow in Section 6 . 


\section{Literature review}

Most of the available literature on autonomous vehicles consider their use and planning in private spaces, such as indoor warehouses. for a recent review, the reader is directed to (Flämig, 2016). Regarding the use of autonomous vehicles in public spaces, only a few studies are available, including one by Fagnant and Kockelman (2014) on the impact of autonomous vehicles on the environment, and another one by de Almeida Correia and van Arem (2016) on the impact of self-driving family vehicles on urban mobility. When it comes to ATs, there seems to exist no study on route and speed optimization assuming the use of AT technology.

In this section, we review some of the relevant existing literature of the PRP and its variants, given the similarities to the problem studied in this paper. Our study contains also stochastic travel times within a VRP framework, therefore it can also be viewed as a natural extension of the VRP with stochastic travel times. Therefore, we also review the relevant literature on this problem. For recent reviews of the literature on green road freight transportation, we refer the reader to Demir et al. (2014b) where the authors review gas emissions models, and to Lin et al. (2014), Eglese and Bektaş (2014) and Bektaş et al. (2016) which are surveys of green VRPs. For a wider and comprehensive overview of the green transportation, including air and maritime transportation, we refer the reader to Psaraftis (2016).

\subsection{The Pollution-Routing problem}

In the original paper of Bektaş and Laporte (2011), the PRP makes use of the comprehensive fuel consumption model proposed by Scora and Barth (2006), and is modeled through a non-linear mathematical formulation in which the decision variables correspond to the selection of routes and speeds. Non-linear constraints are linearized using speed discretization. The authors presented extensive computational results on instances involving up to 20 customers and showed that $\mathrm{CO}_{2}$ emissions could be reduced by up to $8 \%$ by solving a comprehensive model that accounts for pollution. Instead of discretizing speed, Fukasawa et al. (2016) tackled the PRP with continuous speed and put forward two arc-based mixed-integer convex optimization models that can be solved as disjunctive convex programs, and can handle instances with up to 25 customers. Dabia et al. (2016) described a branch-and-price algorithm for the PRP based on a column generation mechanism in which the master problem is a set partitioning problem. The pricing problem is solved by means of modification of a labeling algorithm for the elementary shortest path problem with resource constraints. The 
algorithm can optimally solve all instances with 10 customers, as well as some instances from the PRP-lib with 15 and 20 customers.

Demir et al. (2012) developed an adaptive large neighborhood search (ALNS) metaheuristic for jarger PRP instances which embeds an adaptation of the speed optimization algorithm of Norstad

et al. (2011). The algorithm provided solutions for realistic instances with up to 200 customers within just over 10 minutes. Kramer et al. (2015) later proposed a matheuristic that combines an iterative local search, based on the randomized variable neighborhood descent heuristic of Subramanian et al. (2010), with a speed optimization algorithm and an exact method for a set partitioning problem. When compared with the ALNS of Demir et al. (2012), their matheuristic yielded better quality solutions on all instances with 100 and 200 customers.

Franceschetti et al. (2013) studied a version of the PRP with time-dependent speeds which assumes two periods, one characterized by a congestion speed, and the other when a vehicle can travel at an optimized free-flow speed. The authors used the emissions model of Scora and Barth (2006) to compute fuel consumption. In this problem, service at customers must start within specified time windows, and it is possible for a vehicle to wait at customer after completing service to avoid congestion further along the route. The authors also studied a special case of the problem with a single customer and analytically characterized optimal solutions. They described a speed optimization algorithm for the problem and solved to optimality instances with up to 20 customers.

Qian and Eglese (2014) tackled the problem of minimizing fuel consumption under time-varying speeds. The authors considered both vehicle routes and speeds as decision variables. They proposed two algorithms based on dynamic programming, one of which is exact and the other is heuristic, and tested both on a realistic 14-customer instance. Their results showed that the heuristic is much faster than the exact method, taking five minutes instead of 12 hours. The same authors Qian and Eglese, 2016) later proposed a combined tabu search and column generation algorithm for this problem, based on an earlier algorithm by Prescott-Gagnon et al. (2009) for the VRP with time windows. The algorithm was tested on a real instance with 60 customers and one depot, and the results indicated about three percent savings in GHG emissions compared with a time-minimizing objective.

Maden et al. (2010) considered the VRP with time windows and time-dependent speeds, and developed a tabu search heuristic in which the objective is to minimize the total time spent by the vehicles. The authors showed that up to $7 \%$ of $\mathrm{CO}_{2}$ emissions can be saved on the instances tested. 


\subsection{Vehicle routing problems with stochastic travel times}

The most common sources of uncertainty in stochastic vehicle routing are customer availability, customer demand, service times and travel times (Gendreau et al., 2016). In these variants, some

$$
\text { times were significantly faster than for the } \mathrm{A}^{*} \text { algorithm. }
$$

Eshtehadi et al. (2017) considered the PRP under demand and travel time uncertainty. The authors focused on stochastic customer demand and proposed three mathematical models based on a robust optimization approach. The authors defined a hard-worst case (HWC), a soft-worst case their robustness to uncertainty. Travel time uncertainty is handled by considering a deterministic congestion level. The results showed that considering robust optimization technique to provide 
reliable solutions may result in 30, 50 and 60 liters of additional fuel consumption for 10-, 15- and 20-node instances respectively.

Huang et al. (2017) considered the Time-Dependent VRP (TDVRP) with path flexibility. As opposed to considering a simple path between a pair of customers, the authors start first by determining the set of time-dependent shortest paths on the network using a modified version of Dijkstra's algorithm. The authors describe a mathematical model to the TDVRP with pathflexibility and a two-stage stochastic program to solve a stochastic variant in which speeds on arcs are represented by discrete scenarios. The first-stage decisions relate to customer sequences and the second-stage decisions relate to the selection of paths. Instances with 30 nodes were solved by the deterministic version, and instances with 10 nodes and three discrete scenarios were solved by the stochastic program. The deterministic equivalent of the stochastic PRP with path flexibility yielded better results than solving a standard TDVRP.

\subsection{Relationship with the state of knowledge}

Although the papers just reviewed share some similarities with the problem we introduce here, they exhibit two fundamental differences with respect to the types of decisions and the cost components. The first of these is vehicle speed. The authors of the above papers argue that speed cannot be controlled in urban areas and vehicles must follow the traffic speed. In our study, we consider an intercity travel setting, such as highways, where autonomous trucks have control over the speed. From a methodological point of view, modeling speed as decision variable is challenging due to the non-linearity it entails. The second fundamental difference relates to the cost. In particular, we consider driver wage as part of the objective function. While this may seem a relatively straightforward modification of the objective function, it does make a difference since it has been shown that the largest component of the PRP objective function is the driver's wage (Bektaş and Laporte, 2011). Table 1 summarizes the differences between the papers cited above and our paper.

Our work builds on and contributes to the existing body of work in a number of aspects. First, we continue to treat speed as a decision variable, as was done in the PRP and other relevant studies reviewed in Section 2.1 (e.g., Demir et al., 2012, Fukasawa et al., 2016). However, we break away from the main assumption made in those references in that the vehicle speed is bounded by a constant value. One novel aspect of our contribution is to restrict the vehicle speed on each arc by a 


\begin{tabular}{|c|c|c|c|c|c|c|c|c|}
\hline \multirow[t]{2}{*}{ Paper } & \multicolumn{2}{|c|}{ Objective function } & \multirow{2}{*}{$\begin{array}{c}\text { Time } \\
\text { dependency }\end{array}$} & \multirow{2}{*}{$\begin{array}{c}\text { Speed } \\
\text { decisions }\end{array}$} & \multirow{2}{*}{$\begin{array}{c}\text { Path } \\
\text { flexibility }\end{array}$} & \multirow{2}{*}{$\begin{array}{c}\text { Time } \\
\text { windows }\end{array}$} & \multicolumn{2}{|c|}{ Solution method } \\
\hline & Cost & Fuel & & & & & Exact & Heuristic \\
\hline Ehmke et al. (2016) & - & $\checkmark$ & $\checkmark$ & - & - & - & - & $\checkmark$ \\
\hline Hwang and Ouyang (2015) & $\checkmark$ & $\checkmark$ & - & - & $\checkmark$ & - & $\checkmark$ & $\checkmark$ \\
\hline Eshtehadi et al. (2017) & - & $\checkmark$ & - & $\checkmark$ & - & $\checkmark$ & $\checkmark$ & - \\
\hline Huang et al. (2017) & - & $\checkmark$ & $\checkmark$ & - & $\checkmark$ & - & $\checkmark$ & $\checkmark$ \\
\hline This paper & $\checkmark$ & $\checkmark$ & - & $\checkmark$ & - & $\checkmark$ & $\checkmark$ & - \\
\hline
\end{tabular}

Table 1: Comparison of the problem characteristics studied in this paper and related papers

stochastic parameter, the value of which depends on a future realization of a scenario that prescribes the traffic speeds. This makes for a more realistic treatment of the problem by reflecting part of the uncertainty of the traffic conditions in the relevant models. Second, we assume that the travel times are stochastic by following a line of analysis similar to that of the references reviewed in Section 2.2. However, whereas the references cited above treat the travel time on an arc as an uncertain input parameter, we model it as a function of the speed chosen to traverse that arc, implying that travel time itself is effectively a decision variable, but one that is subject to uncertainty. The latter aspect of our contribution is therefore different and unique. While the robust approach proposed by Eshtehadi et al. (2017) for the pollution-routing problem considers speed as a decision variable, it tends to be over-conservative since it assumes the realization of a worst-case scenario. Robust solutions are therefore costlier than those produced by the stochastic programming methods we advocate in this paper. Finally, we resort to two-stage stochastic programming formulations with recourse as the modeling framework, for the very reason that this approach is shown to be a suitable way to formulate such problems (e.g., Laporte et al., 1992), and with an objective function that minimizes the expected cost (e.g., Laporte et al., 1992, Taş et al., 2013).

\section{Technical background information}

In our study, we use the same consumption model as in Bektaş and Laporte (2011), which we now describe. From this model and by using an illustrative example, we show also the impact that stochastic travel times may have on the amount of emissions. We then propose two ways of modeling our problem. 


\subsection{Calculation of fuel consumption and emissions}

There exist numerous emission models, which were reviewed and compared in Demir et al. (2014b). These models vary with respect to the input parameters and level of detail, but most use non-linear functions of vehicle speed. Such functions generally have a convex U-shaped form, which can be optimized to yield a speed minimizing fuel consumption and emissions. Our emissions model is an instantaneous model proposed by Barth and Boriboonsomsin (2008), which estimates the fuel consumed $F_{r}$ per second based on the function

$$
F_{r}=\varepsilon(k \Omega V+P / \eta) / \kappa,
$$

where $\eta$ and $\kappa$ are constants related to diesel fuel typically used by delivery vehicles, $\varepsilon$ is fuel-toair mass ratio, $k, \Omega$ and $V$ are the engine friction factor, engine speed and engine displacement respectively. Furthermore, $P$ is the engine power output per second (in $\mathrm{kW}$ ) and is calculated as

$$
P=P_{\text {tract }} / \eta_{t f}+P_{\text {acc }}
$$

where $\eta_{t f}$ is the vehicle drive train efficiency, and $P_{\text {acc }}$ is the engine power demand associated with running losses of the engine and the operation of vehicle accessories (i.e., air conditioning). In our study, we assume that $P_{\text {acc }}$ is equal to zero. The parameter $P_{\text {tract }}$ is the total tractive power requirements (in $\mathrm{kW}$ ) placed on the wheels:

$$
P_{\text {tract }}=\left(\Lambda \tau+\Lambda g \sin \theta+0.5 C_{d} \rho \Gamma v^{2}+\Lambda g C_{r} \cos \theta\right) v / 1000
$$

with $\Lambda$ and $v$ are the vehicle weight $(\mathrm{kg})$ and speed $(\mathrm{m} / \mathrm{s})$ respectively, $\tau$ is the acceleration $\left(\mathrm{m} / \mathrm{s}^{2}\right)$, $\theta$ is the road angle, $g$ is the gravitational constant $\left(\mathrm{m} / \mathrm{s}^{2}\right)$, and $C_{d}$ and $C_{r}$ are the coefficients of the aerodynamic drag and rolling resistance, respectively. Finally, $\rho$ is the air density $\left(\mathrm{kg} / \mathrm{m}^{3}\right)$, and $\Gamma$ is the frontal surface area of the vehicle $\left(\mathrm{m}^{2}\right)$. For a given $\operatorname{arc}(i, j)$ of length $d$ and assuming that all parameters remain constant except for the vehicle speed, we can express fuel consumption in liters (L) as

$$
\begin{aligned}
F(v) & =k \Omega V \lambda d / v \\
& +\left(w \alpha v+\alpha f v+\beta v^{3}\right) \lambda \gamma d / v,
\end{aligned}
$$

where $\lambda$ and $\gamma$ are constants defined as $\lambda=\varepsilon / \kappa \Psi$, where $\Psi$ is the conversion factor of fuel from $(\mathrm{g} / \mathrm{s})$ to $(\mathrm{L} / \mathrm{s})$, and $\gamma=1 /\left(1000 \eta_{t f} \eta\right)$. Furthermore, if $\Lambda$ is the total weight of vehicle between node $i$ and 
$j$, then $\Lambda=w+f$, where $w$ is the weight of an empty vehicle (curb weight), and $f$ is the vehicle load. Let $\alpha=\tau+g \sin \theta+g C_{r} \cos \theta$ be a vehicle-arc specific constant and $\beta=0.5 C_{d} \rho \Gamma$ be a vehicle specific constant. We omit indices $(i, j)$ on the variables $v, d, f$, and $\alpha$ to simplify the presentation.

\subsection{The impact of travel speed uncertainty on emissions}

Traffic congestion is one of the main factors preventing vehicles from driving at an optimal speed. It can be caused by accidents, bad weather, or simply the traffic level in the network. Congestion is typically characterized by low speeds and regular start-and-stops. As a result, it drastically increases fuel consumption compared with the non-congestion case.

We now illustrate how congestion may impact the routing decisions on a four-node instance corresponding to four British cities, where the fictional demand of each customer and the service times for this instance are given in Table 2. The time windows of the customers (in minutes) are shown in Figure 1a The distance matrix of Table 3 shows the distances $(\mathrm{m})$ between the nodes of the network.

\begin{tabular}{c|c|c|c}
\hline Node index & City & Demand $(\mathrm{kg})$ & Service time (min) \\
\hline 0 & Kingston upon Hull (depot) & - & - \\
1 & Pocklington & 721 & 24 \\
2 & Brough & 814 & 27 \\
3 & Selby & 620 & 21 \\
\hline
\end{tabular}

Table 2: Demand and service time data

\begin{tabular}{c|cccc}
\hline Node index & 0 & 1 & 2 & 3 \\
\hline 0 & 0 & 41150 & 25680 & 54200 \\
1 & 40660 & 0 & 51980 & 32800 \\
2 & 25010 & 51780 & 0 & 61520 \\
3 & 54270 & 32750 & 61560 & 0 \\
\hline
\end{tabular}

Table 3: Distance matrix for the example of Table 2

We assume that the traffic speeds on all the arcs of the network is not known in advance, but is 
instead a discrete random variable referred to as a scenario. In this example, we consider three scenarios $S_{1}, S_{2}$ and $S_{3}$ with respective probabilities $33 \%, 33 \%$ and $34 \%$. Below, we show the matrices representing the three scenarios $S_{1}, S_{2}$ and $S_{3}$ for this example where each entry $(i, j)$ in a matrix represents the traffic speeds $(\mathrm{km} / \mathrm{h})$ on each $\operatorname{arc}(i, j)$. The scenarios $S_{1}, S_{2}$ and $S_{3}$ are derived from a probability distribution discussed further in Section 5.1 .

$$
S_{1}=\left(\begin{array}{cccc}
0 & 22 & 28 & 35 \\
21 & 0 & 40 & 23 \\
67 & 56 & 0 & 59 \\
46 & 37 & 29 & 0
\end{array}\right) \quad S_{2}=\left(\begin{array}{cccc}
0 & 59 & 48 & 60 \\
31 & 0 & 53 & 28 \\
43 & 46 & 0 & 33 \\
41 & 70 & 30 & 0
\end{array}\right) \quad S_{3}=\left(\begin{array}{cccc}
0 & 22 & 21 & 50 \\
39 & 0 & 60 & 23 \\
37 & 21 & 0 & 70 \\
33 & 29 & 58 & 0
\end{array}\right) .
$$

The problem is to find an optimal route and the optimal speeds on each arc, where the chosen speeds are constrained by the chosen speeds. One way to solve the problem is by creating an average scenario $S_{a v}$ where the traffics speeds on each arc $(i, j)$ is the average of all traffic speeds over all scenarios, taking into account their respective probability and solve a deterministic PRP. $S_{a v}$ is represented by a matrix where each item $S_{a v}(i, j)=0.33 S_{1}(i, j)+0.33 S_{2}(i, j)+0.34 S_{3}(i, j)$.

Figure 1 shows two optimal solutions obtained by using $£ 1.4$ as the combined unit cost of fuel (per litre) and $\mathrm{CO}_{2}$, and $£ 10$ as the hourly driver cost. The solution shown in Figure $1 \mathrm{a}$ is obtained by solving the deterministic PRP where the traffic speeds are prescribed by the matrix $S_{a v}$, and are imposed as upper limits on the speeds that can be chosen on each arc. The optimal speeds are shown on the arcs. The cost of this solution is £99.96, and its expected value when calculated under the realization of $S_{1}, S_{2}$ and $S_{3}$, is equal to $£ 121.58$. However, this approach is suboptimal as it does not fully utilize all the available information. In contrast, Figure $1 \mathrm{~b}$ shows the solution generated using stochastic programming where the three scenarios $S_{1}, S_{2}$ and $S_{3}$ are explicitly taken into account during the solution process. This solution has an expected cost equal to £106.70, which represents a saving of $12.26 \%$ over the deterministic. The way in which stochastic programming is used to produce the latter solution will be further explained in Section 4.2.1. 


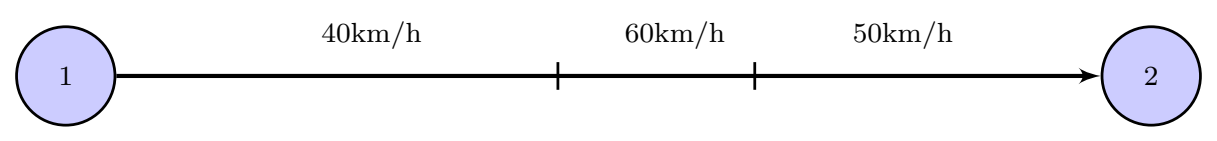

Figure 2: Example of speed variability

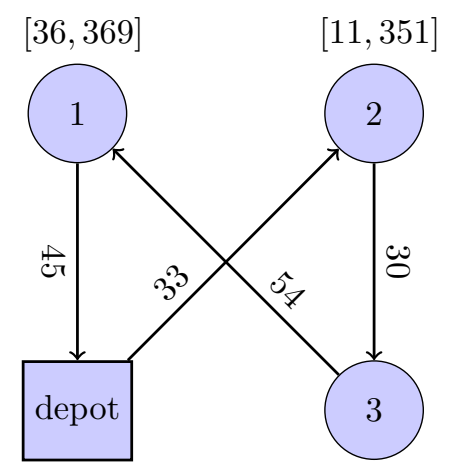

$[17,340]$

(a) The solution obtained by considering $S_{a v}$

(b) The solution obtained by considering the scenarios $\left(S_{1}, S_{2}\right.$ and $\left.S_{3}\right)$

Figure 1: Two solutions of the four-node instance

Variability of traffic speed on a given intercity road can be better represented by dividing an arc between two customers into smaller segments. The traffic speed will then be defined for every segment, where for each segment an optimal speed can be prescribed. To illustrate, we consider the example in Figure 2, which shows an arc $(1,2)$ of $100 \mathrm{~km}$ length, on which we assume the traffic speed to be $50 \mathrm{~km} / \mathrm{h}$. Suppose now that further information is available about the variability of traffic speeds on this arc such that it can be divided into three segments of $50 \mathrm{~km}, 20 \mathrm{~km}$ and $30 \mathrm{~km}$, where the traffic speed on each segment is $40 \mathrm{~km} / \mathrm{h}, 60 \mathrm{~km} / \mathrm{h}$ and $50 \mathrm{~km} / \mathrm{h}$, respectively. If we consider a vehicle traveling at $50 \mathrm{~km} / \mathrm{h}$, assuming a constant traffic speed throughout the arc, the combined cost of fuel, $\mathrm{CO}_{2}$ and driver is £34. If however, we consider the three segments and that the vehicle travels at the traffic speed on each of the segments, the total cost is $£ 37$, which is more accurate, but is at the expense of a $9 \%$ increase. 

travel on each arc of the routes. No further action can be taken to change the routes and speeds to improve the solution after this point. We will refer to this strategy as "one-route, one-speed" (OR-OS).

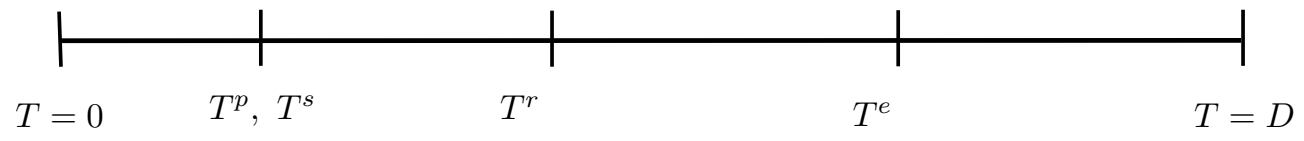

Figure 3: Time-line of the first strategy decision making process

Under the second strategy, we also assume that the scenarios are revealed after the routes have 
speeds, as shown in Figure 4. This situation occurs in practice when the vehicles must be loaded the day before the routing plans are operationalized, and the information about traffic speeds is revealed before the start of routing. In this case, the routes cannot be redesigned assuming that the goods are already loaded in the vehicles. We will refer to this strategy as "one-route, several speeds" (OR-SS). This strategy is unlikely to be applied in practice given that the information required is almost impossible to acquire. However, the aim of considering such a strategy is to assess the value of the added information and the savings that it can provide.

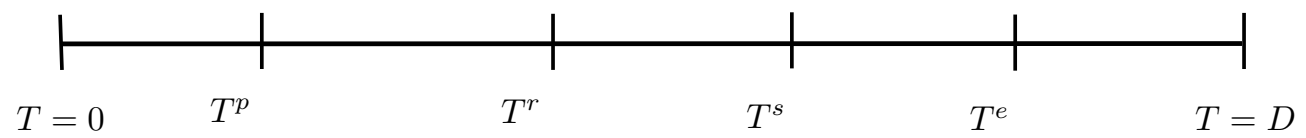

Figure 4: Time-line of the second strategy decision making process

\section{Problem Description and Mathematical Models}

The problem studied in this paper is similar to the PRP, where the traffic speeds constitute the upper bounds on the maximum speed that can be attained on each leg of the route are not known with certainty at the time of route planning. Instead, they are described by a discrete and finite set of scenarios. The problem involves determining a set of vehicle routes and speeds on each leg of the routes, as in the PRP. However, upon realization of the random variables, the optimal speeds may no longer be feasible with respect to the traffic speeds, in which case delays may occur in arriving at customer locations. The objective is to minimize the expected cost of the total fuel consumption, emissions, drivers and delays. The problem is cast as a two-stage stochastic programming problem (Birge and Louveaux, 2011), for which we define two variants. The first variant corresponds to the OR-OS strategy, where the first-stage decisions involve determining the routes and the arc speeds. The second-stage decisions are the speed reductions inferred by the traffic speeds, which translate into possible delays. The second variant is based on the OR-SS strategy, where the first stage decisions are restricted to planning a route for each vehicle, and the second stage variables correspond to computing the speeds on each route, separately for each scenario. In what follows, we describe mathematical models associated with the two problem variants just described. 


\subsection{Notations Common to the Two Models}

The stochastic models presented in this paper are based on the deterministic mathematical model of the classical PRP proposed by Demir et al. (2012). The problem is defined on a complete graph $G=(N, A)$, where $N=\{0,1, \ldots, n\}$ is the node set, and $A=\{(i, j) ; i, j \in N, i \neq j\}$ is the arc set, where each $\operatorname{arc}(i, j)$ has $\left|L_{i j}\right|$ segments of length $d_{i j l}, L_{i j}=\left\{1, \ldots, l, \ldots,\left|L_{i j}\right|\right\}$. Node 0 represents the depot at which $m$ vehicles of capacity $Q$ are based, and $N_{0}=N \backslash\{0\}$ is the set of customers. Each customer $i \in N_{0}$ has a demand $q_{i}>0$, which should start to be delivered within the time window $\left[a_{i}, b_{i}\right]$, and incurs a service time $c_{i}$. The decision variables are defined as follows: $x_{i j}$ is a binary variable equal to 1 if and only if a vehicle visits customer $j$ immediately after customer $i$. A vehicle travels at speed $v_{i j l}$ on segment $l \in L_{i j}$ of $\operatorname{arc}(i, j)$, subject to a lower bound $v^{l b}$ and an upper bound $v^{u b}$ that correspond to legal limits on speed. Under uncertainty, a traffic speed $u_{i j l}^{s}$ is defined on segment $l \in L_{i j}$ of arc $(i, j)$ for each scenario $s \in S$ which is the maximum attainable speed on that arc. The continuous variable $f_{i j}$ is the amount of commodity carried on the vehicle on $\operatorname{arc}(i, j) \in A$. The continuous variable $y_{i}$ is the time at which service begins at customer $i \in N_{0}$, and the continuous variable $\delta_{i}$ is the the cumulative time spent on the route whose last customer is $i$.

\subsection{One-Route, One-Speed Strategy}

Under the OR-OS strategy, we assume that the decisions concerning routes and speeds are made simultaneously, and prior to the realization of the random variables. These two sets of decisions are made at the first stage and cannot subsequently be changed. However, if $v_{i j l}^{*}>u_{i j l}^{s}$, a vehicle will not be able to drive at speed $v_{i j l}^{*}$ chosen on a specific segment $l$ of arc $(i, j)$ for a particular a scenario $s \in S$. In this case, the recourse action is to reduce the speed by at least $v_{i j l}^{*}-u_{i j l}^{s}$ in order for the solution to be feasible. To this end, we introduce a speed reduction recourse variable $S R_{i j l}^{s}$ to ensure the feasibility of solutions independently of random variables realizations. The scenarios also imply that some customers may not be served within their time window. Therefore, the time window requirements will be treated as soft constraints, in which case, the delay for the start of service at customer $i$ will be represented by a recourse variable $l_{i}^{s}$ in the second stage, and penalized by using a unit delay cost $e$.

It is important to observe that speed reduction recourse variables are needed in the computation of delays and routes durations, but are not explicitly penalized in the objective function. They are 
only implicitly penalized because the vehicle will have to travel at a speed different from the optimal speed, which will increase the cost accordingly.

The speed chosen on an arc will affect the time $y_{i}$ at which service starts at node $i$, and hence it will be different from one scenario to another. For this reason, this time will be represented by a scenario-dependent variable $y_{i}^{s}$. This variable captures the fact that a vehicle can be late to serve a customer in one scenario, but on time in a another one. The same reasoning applies to the variables representing the overall time spent on a route $\delta_{i}$ which will now be $\delta_{i}^{s}$. We define $M$ as a large enough constant. The mathematical model is described as follows:

$$
\begin{aligned}
\text { Minimize } & \sum_{(i, j) \in A} \sum_{l \in L_{i j}} w f_{c} \gamma \lambda \alpha_{i j} d_{i j l} x_{i j}+\sum_{(i, j) \in A} \sum_{l \in L_{i j}} f_{c} \gamma \lambda \alpha_{i j} f_{i j} d_{i j l} \\
& +\sum_{s \in S} p_{s}\left(f_{c} k \Omega V \lambda \sum_{(i, j) \in A} \sum_{l \in L_{i j}} d_{i j l} /\left(v_{i j l}-S R_{i j l}^{s}\right)\right. \\
& +f_{c} \beta \gamma \lambda \sum_{(i, j) \in A} \sum_{l \in L_{i j}} d_{i j l}\left(v_{i j l}-S R_{i j l}^{s}\right)^{2} \\
& +\sum_{j \in N_{0}} f_{d} \delta_{j}^{s} \\
& \left.+\sum_{j} e l_{j}^{s}\right)
\end{aligned}
$$

subject to

$$
\begin{array}{ll}
\sum_{j \in N} x_{0 j}=m & \\
\sum_{j \in N} x_{i j}=1 & i \in N_{0} \\
\sum_{i \in N} x_{i j}=1 & j \in N_{0} \\
\sum_{j \in N} f_{j i}-\sum_{j \in N} f_{i j}=q_{i} & i \in N_{0} \\
q_{j} x_{i j} \leq f_{i j} \leq\left(Q-q_{i}\right) x_{i j} & (i, j) \in A \\
y_{i}^{s}-y_{j}^{s}+c_{i}+\sum_{l \in L_{i j}} d_{i j l} /\left(v_{i j l}-S R_{i j l}^{s}\right) \leq M\left(1-x_{i j}\right) & i \in N, j \in N_{0}, i \neq j, s \in S \\
y_{j}^{s}-c_{j}-\delta_{j}^{s}+\sum_{l \in L_{i j}} d_{j 0 l} /\left(v_{j 0 l}-S R_{j 0 l}^{s}\right) M\left(1-x_{j 0}\right) & j \in N_{0}, s \in S \\
a_{j} \leq y_{j}^{s} \leq b_{j}+l_{j}^{s} & j \in N_{0}, s \in S
\end{array}
$$




$$
\begin{array}{ll}
v_{i j l}-S R_{i j l}^{s} \leq u_{i j l}^{s} & (i, j) \in A, l \in L_{i j}, s \in S \\
v_{i j l}-S R_{i j l}^{s} \geq v^{l b} x_{i j} & (i, j) \in A, l \in L_{i j}, s \in S \\
f_{i j} \geq 0 & (i, j) \in A \\
y_{i}^{s} \geq 0 & i \in N_{0}, s \in S \\
S R_{i j}^{s} \geq 0 & (i, j) \in A, s \in S \\
v^{l b} x_{i j} \leq v_{i j l} \leq v^{u b} x_{i j} & (i, j) \in A, l \in L_{i j} \\
x_{i j} \in\{0,1\} & (i, j) \in A .
\end{array}
$$

The first term of the objective function computes the cost of the solution that can be attributed to vehicle weight and load. Similarly, the terms $(7)-10$ represent the expected cost as a function of speeds, delays at customers, and driving time. Constraints (11) ensure that all vehicles leave the depot. Constraints 12 - 13 state that each customer must be visited exactly once. Constraints

(14) and (15) define the flow on each arc. Constraints (16)-17) compute the arrival time at each customer $j \in N_{0}$ in each scenario $s \in S$, (18) calculate the delay at customers, (19) ensure that the speed used in a scenario after any reduction is lower than the traffic speed in that scenario. Constraints 20 ensure that the speeds chosen are at least $v^{l}$ if the arc is in the route of a vehicle.

Due to the terms (7), 8), (16) and (17), the model described above is non-linear. In the following, we present two different ways of linearizing these terms.

\subsubsection{Discrete Speeds and Discrete Recourse}

In order to linearize the non-linear terms, we adopt the same strategy as in Bektaş and Laporte (2011), which consists of discretizing the speed variables. We will also discretize the recourse variables. The speeds are represented by a set $R=\{1, \ldots, r, \ldots\}$ of discrete levels. A binary variable $z_{i j l}^{r}$ is equal to 1 if and only if the $r^{\text {th }}$ speed level $v^{r}$ is chosen for the vehicle traveling on segment $l \in L_{i j}$ of $\operatorname{arc}(i, j)$. We note that $v^{1}=v^{l b}$ and $v^{|R|}=v^{u b}$. The same reasoning applies to the discretized recourse. We define a set $|T|$ of recourse levels $T=\{1, \ldots, t, \ldots\}$, and a binary variable $\alpha_{i j l}^{t s}$ is equal to 1 if and only if the level $t$ of speed reduction level $\psi^{t}$ is chosen for the vehicle traveling on segment $l \in L_{i j}$ of $\operatorname{arc}(i, j)$ in scenario $s \in S$, with $\psi^{1}=0$ and $\psi^{|T|}=v^{u b}-v^{l b}$. We also introduce also $M$ as a large constant. The discretized mathematical model is described as follows.

$$
\operatorname{Minimize} \sum_{(i, j) \in A} \sum_{l \in L_{i j}} w f_{c} \gamma \lambda \alpha_{i j} d_{i j l} x_{i j}+\sum_{(i, j) \in A} \sum_{l \in L_{i j}} f_{c} \gamma \lambda \alpha_{i j} d_{i j l} f_{i j}
$$




$$
\begin{aligned}
& +\sum_{s \in S} p_{s}\left(f_{c} k \Omega V \lambda \sum_{(i, j) \in A} \sum_{l \in L_{i j}} d_{i j l}\left(\sum_{r \in R} \sum_{t \in T} z_{i j l}^{r} \alpha_{i j l}^{t s} /\left(v^{r}-\psi^{t}\right)\right)\right. \\
& +f_{c} \beta \gamma \lambda \sum_{(i, j) \in A} \sum_{l \in L_{i j}} d_{i j l} \sum_{r \in R} \sum_{t \in T} z_{i j}^{r} \alpha_{i j l}^{t s}\left(v^{r}-\psi^{t}\right)^{2} \\
& +\sum_{j \in N_{0}} f_{d} \delta_{j}^{s} \\
& \left.+\sum_{j} e l_{j}^{s}\right)
\end{aligned}
$$

subject to

(11)-15, , 18, 21, 22,

$$
\begin{array}{ll}
y_{i}^{s}-y_{j}^{s}+c_{i}+\sum_{l \in L_{i j}} d_{i j l} \sum_{r \in R} \sum_{t \in T} z_{i j l}^{r} \alpha_{i j l}^{t s} /\left(v^{r}-\psi^{t}\right) \leq M\left(1-x_{i j}\right) & i \in N, \\
y_{j}^{s}-c_{j}-\delta_{j}^{s}+\sum_{l \in L_{i j}} d_{j 0 l} \sum_{r \in R} \sum_{t \in T} z_{j 0 l}^{r} \alpha_{j 0 l}^{t s} /\left(v^{r}-\psi^{t}\right) \leq M\left(1-x_{j 0}\right) & j \in N_{0}, i \neq j, s \in S \\
\alpha_{i j l}^{1 s} \geq\left(u_{i j l}^{s}-\sum_{r \in R} z_{i j l}^{r} v^{r}\right) / M+\left(x_{i j}-1\right) & (i, j) \in A, l \in L_{i j}, s \in S \\
\alpha_{i j l}^{1 s} \leq\left(u_{i j l}^{s}-\sum_{r \in R} z_{i j l}^{r} v^{r}\right) / M+1 & (i, j) \in A, l \in L_{i j}, s \in S \\
\sum_{r \in R} \sum_{t \in T} z_{i j l}^{r} \alpha_{i j l}^{t s}\left(v^{r}-\psi^{t}\right) \leq u_{i j l}^{s} & (i, j) \in A, l \in L_{i j}, s \in S \\
\sum_{r \in R} \sum_{t \in T} z_{i j l}^{r} \alpha_{i j l}^{t s}\left(v^{r}-\psi^{t}\right) \geq v^{1} x_{i j} & (i, j) \in A, l \in L_{i j}, s \in S \\
\sum_{r \in R} z_{i j l}^{r}=x_{i j} & (i, j) \in A, l \in L_{i j} \\
\sum_{t \in T} \alpha_{i j l}^{t s}=x_{i j} & (i, j) \in A, l \in L_{i j}, s \in S \\
\alpha_{i j l}^{t s}, z_{i j l}^{r}, x_{i j} \in\{0,1\} & (i, j) \in A, l \in L_{i j}, \\
& s \in S, r \in R, t \in T .
\end{array}
$$

Constraints (31)-32 compute the arrival time at each customer $j \in N_{0}$ in each scenario $s \in S$. Constraints (33)-(34) ensure that the value of the speed reduction is 0 if the speed chosen is lower than the traffic speed in scenario $s$. Constraints $(35)-(36)$ play the same role as $(19)-(20)$. Finally, constraints 38 ensure that only one recourse is chosen if arc $(i, j)$ is selected. 
This model is still non-linear due to the presence of products of two binary variables in constraints (35) and 36. To linearize these constraints, we introduce a new binary variable $\tau_{i j l}^{r t s}$ equal to 1 if and only if a vehicle travels on segment $l$ of arc $(i, j)$ at speed $v^{r}$ and applies a speed recourse $\psi^{t}$ in scenario $s \in S$. The variables $\tau_{i j l}^{r t s}$ will replace the products $z_{i j l}^{r} \alpha_{i j l}^{t s}$ through the use of the following constraints:

$$
\begin{aligned}
\tau_{i j l}^{r t s} \leq z_{i j l}^{r} & (i, j) \in A, l \in L_{i j}, r \in R, s \in S \\
\tau_{i j l}^{r t s} \leq \alpha_{i j l}^{t s} & (i, j) \in A, l \in L_{i j}, t \in T, s \in S \\
\tau_{i j l}^{r t s} \geq z_{i j l}^{r}+\alpha_{i j l}^{t s}-1 & (i, j) \in A, l \in L_{i j}, r \in R, t \in T, s \in S \\
\tau_{i j l}^{r t s} \in\{0,1\} & (i, j) \in A, l \in L_{i j}, s \in S, r \in R, t \in T .
\end{aligned}
$$

\subsubsection{Discrete Speeds and Continuous Restricted Recourse}

In the previous model, we used discretization to linearize the mathematical program. However, due to the uncertainty about the traffic speeds, using discrete speed reductions may result in poor quality recourse actions. We therefore introduce an alternative modeling scheme based on the use of continuous recourse variables, using same notation as in Section 4.2.1. However, here we make an additional assumption that restricts the value of the recourse action. Recall that $S R_{i j l}^{s}$ is the speed reduction needed on segment $l$ of $\operatorname{arc}(i, j) \in A$ to travel at a speed not exceeding $u_{i j l}^{s}$ in scenario $s \in S$. We now assume that if a speed recourse is needed, then $S R_{i j l}^{s}$, will be exactly equal to $\sum_{r \in R} z_{i j l}^{r} v^{r}-u_{i j l}^{s}$. This assumption is needed to linearize the model, and to have continuous recourse variables. We introduce a binary activation variable $a_{i j l}^{s}$ equal to 1 if and only if a speed reduction is needed on segment $l$ of $\operatorname{arc}(i, j) \in A$ in scenario $s \in S$. The mathematical model is described as follows:

$$
\begin{aligned}
\text { Minimize } & \sum_{(i, j) \in A} \sum_{l \in L_{i j}} w f_{c} \gamma \lambda \alpha_{i j} d_{i j l} x_{i j}+\sum_{(i, j) \in A} \sum_{l \in L_{i j}} f_{c} \gamma \lambda \alpha_{i j} d_{i j l} f_{i j} \\
& +\sum_{s \in S} p_{s}\left(f_{c} k \Omega V \lambda \sum_{(i, j) \in A} \sum_{l \in L_{i j}} d_{i j l}\left(\sum_{r \in R} z_{i j l}^{r} /\left(v^{r}-S R_{i j l}^{s}\right)\right)\right. \\
& +f_{c} \beta \gamma \lambda \sum_{(i, j) \in A} \sum_{l \in L_{i j}} d_{i j l} \sum_{r \in R} z_{i j l}^{r}\left(v^{r}-S R_{i j l}^{s}\right)^{2} \\
& +\sum_{j \in N_{0}} f_{d} \delta_{j}^{s} \\
& \left.+\sum_{j} e l_{j}^{s}\right)
\end{aligned}
$$


subject to

(11)-(15), (18), 21)-23)

$$
\begin{array}{ll}
y_{i}^{s}-y_{j}^{s}+c_{i}+\sum_{l \in L_{i j}} \sum_{r \in R} d_{i j l} z_{i j l}^{r} /\left(v^{r}-S R_{i j l}^{s}\right) \leq M\left(1-x_{i j}\right) & i \in N, j \in N_{0}, i \neq j, s \in S \\
y_{j}^{s}-c_{j}-\delta_{j}^{s}+\sum_{l \in L_{i j}} \sum_{r \in R} d_{j 0 l} z_{j 0 l}^{r} /\left(v^{r}-S R_{i j l}^{s}\right) \leq M\left(1-x_{j 0}\right) & j \in N_{0}, s \in S \\
S R_{i j l}^{s} \geq \sum_{r \in R} z_{i j l}^{r} v^{r}-u_{i j l}^{s} & (i, j) \in A, l \in L_{i j}, s \in S \\
S R_{i j l}^{s} \leq \sum_{r \in R} z_{i j l}^{r} v^{r}-u_{i j l}^{s}+\left(1-a_{i j l}^{s}\right) M & (i, j) \in A, l \in L_{i j}, s \in S \\
S R_{i j l}^{s} \leq a_{i j l}^{s} M & (i, j) \in A, l \in L_{i j}, s \in S \\
a_{i j l}^{s} \geq\left(\sum_{r \in R} z_{i j l}^{r} v^{r}-u_{i j l}^{s}\right) / M & (i, j) \in A, l \in L_{i j}, s \in S \\
a_{i j l}^{s} \leq\left(\sum_{r \in R} z_{i j l}^{r} v^{r}-u_{i j l}^{s}\right) / M+1 & (i, j) \in A, l \in L_{i j}, s \in S \\
a_{i j l}^{s}, z_{i j l}^{r}, x_{i j l} \in\{0,1\} & (i, j) \in A, r \in R \\
\sum_{r \in R} z_{i j}^{r}=x_{i j} & (i, j) \in A .
\end{array}
$$

In the objective function, the term (44) computes the cost of the solution associated to the vehicle weight and load. The terms (45)-(48) compute the expected cost as a function of speeds, delays at customers and drivers' wages. Constraints 49 - 50 define the arrival time at each customer $j \in N_{0}$ in each scenario $s \in S$. Constraints (51)-55) force $S R_{i j l}^{s}$ to be equal to $\sum_{r \in R} z_{i j l}^{r} v^{r}-u_{i j l}^{s}$ if and only if there is a speed reduction.

Due to the presence of the terms (45) and 446) in the objective function, the model is non-linear. In order to linearize these terms, we introduce two sets of new variables $S F_{i j l}^{s}$ and $S S_{i j l}^{s}$. These new variables are such that $S F_{i j l}^{s}=1 / u_{i j l}^{s}-\sum_{r \in R} z_{i j l}^{r} / v^{r}$ if and only if there is a speed reduction. Using the same reasoning, $S S_{i j l}^{s}=\sum_{r \in R} z_{i j l}^{r}\left(v^{r}\right)^{2}-\left(u_{i j l}^{s}\right)^{2}$. Components 45 and 46 then become

$$
\begin{aligned}
& f_{c} k \Omega V \lambda \sum_{(i, j) \in A} \sum_{l \in L_{i j}} d_{i j l}\left(\sum_{r \in R}\left(z_{i j l}^{r} / v^{r}\right)+S F_{i j l}^{s}\right) \\
& f_{c} \beta \gamma \lambda \sum_{(i, j) \in A} \sum_{l \in L_{i j}} d_{i j l}\left(\sum_{r \in R} z_{i j l}^{r}\left(v^{r}\right)^{2}-S S_{i j l}^{s}\right) .
\end{aligned}
$$


We also replace constraints $449-(53)$ with constraints $60--695$ :

$$
\begin{array}{ll}
y_{i}^{s}-y_{j}^{s}+c_{i}+\sum_{l \in L_{i j}} \sum_{r \in R} d_{i j l}\left(\sum_{r \in R}\left(z_{i j l}^{r} / v^{r}\right)+S F_{i j l}^{s}\right) \leq M\left(1-x_{i j}\right) & i \in N, j \in N_{0}, i \neq j, \\
y_{j}^{s}-c_{j}-\delta_{j}^{s}+\sum_{l \in L_{i j}} \sum_{r \in R} d_{j 0 l}\left(\sum_{r \in R}\left(z_{j 0 l}^{r} / v^{r}\right)+S F_{j 0 l}^{s}\right) \leq M\left(1-x_{j 0}\right) & j \in N_{0}, s \in S \\
S F_{i j l}^{s} \geq 1 / u_{i j l}^{s}-\sum_{r \in R} z_{i j l}^{r} / v^{r}-\left(1-x_{i j}\right) M & (i, j) \in A, l \in L_{i j}, s \in S \\
S F_{i j l}^{s} \leq 1 / u_{i j l}^{s}-\sum_{r \in R} z_{i j l}^{r} / v^{r}+\left(2-a_{i j l}^{s}-x_{i j}\right) M & (i, j) \in A, l \in L_{i j}, s \in S \\
S F_{i j l}^{s} \leq a_{i j l}^{s} M & (i, j) \in A, l \in L_{i j}, s \in S \\
S S_{i j l}^{s} \geq \sum_{r \in R} z_{i j l}^{r}\left(v^{r}\right)^{2}-\left(u_{i j l}^{s}\right)^{2}-\left(1-x_{i j}\right) M & (i, j) \in A, l \in L_{i j}, s \in S \\
S S_{i j l}^{s} \leq \sum_{r \in R} z_{i j l}^{r}\left(v^{r}\right)^{2}-\left(u_{i j l}^{s}\right)^{2}+\left(2-a_{i j l}^{s}-x_{i j}\right) M & (i, j) \in A, l \in L_{i j}, s \in S \\
S S_{i j l}^{s} \leq a_{i j l}^{s} M & (i, j) \in A, l \in L_{i j}, s \in S \\
S F_{i j l}^{s} \geq 0 & (i, j) \in A, l \in L_{i j}, s \in S \\
S S_{i j l}^{s} \geq 0 & (i, j) \in A, l \in L_{i j}, s \in S .
\end{array}
$$

We now present a proposition that establishes the equivalence between the non-linear and the linearized formulations of the discrete speed and continuous recourse.

Proposition 1. Let $v_{N L P}^{*}$ be the optimal value and $\left(x^{*}, z^{*}, k^{*}, S R^{*}\right)$ be an optimal solution of the non-linear formulation. Let $v_{L P}^{*}$ be the optimal value and $\left(x^{*}, z^{*}, k^{*}, S S^{*}, S F^{*}\right)$ be an optimal solution of the linearized formulation. If $445-(46)$ and $449-(53)$ are replaced by $(58)-(59)$ and $(60)-$ 69) respectively, then $v_{N L P}^{*}=v_{L P}^{*}$ and the optimal solutions coincide with respect to $\left(x^{*}, z^{*}, k^{*}\right)$.

Proof. To prove the validity of Proposition 1, we compute the value of the two original terms in the objective functions, and the terms that replace them in the linearized version. The value of these terms needs to be the same. For a given $\operatorname{arc}(i, j) \in A$, there are four cases:

1. There is no speed violation on $(i, j)$ : 
(a) non-linear model: Due to (54)-55) $a_{i j l}^{s}=0$, hence, due to [53), $S R_{i j l}^{s}=0$ for all $s \in S$. Therefore the values of $45-(46)$ are as follows :

- $d_{i j l} \sum_{r \in R} z_{i j l}^{r} /\left(v^{r}-S R_{i j l}^{s}\right)=d_{i j l} \sum_{r \in R} z_{i j l}^{r} / v^{r}=d_{i j l} / v^{r}$ if $z_{i j l}^{r}=1$, and 0 otherwise, - $d_{i j l} \sum_{r \in R} z_{i j l}^{r}\left(v^{r}-S R_{i j l}^{s}\right)^{2}=d_{i j l} \sum_{r \in R} z_{i j l}^{r}\left(v^{r}\right)^{2}=d_{i j l}\left(v^{r}\right)^{2}$ if $z_{i j l}^{r}=1$, and 0 otherwise.

(b) linearized model: Due to 54 55 $a_{i j l}^{s}=0$, hence, due to (64), $S F_{i j l}^{s}=0$ and due to (67) $S S_{i j l}^{s}=0$ for all $s \in S$. Therefore the value of $58-(59)$ are as follows:

- $d_{i j l} \sum_{r \in R}\left(z_{i j l}^{r} / v^{r}\right)+S F_{i j l}^{s}=d_{i j l} \sum_{r \in R} z_{i j l}^{r} / v^{r}=d_{i j l} / v^{r}$ if $z_{i j l}^{r}=1$, and 0 otherwise, - $d_{i j l} \sum_{r \in R} z_{i j l}^{r}\left(v^{r}\right)^{2}-S S_{i j l}^{s}=d_{i j l} \sum_{r \in R} z_{i j l}^{r}\left(v^{r}\right)^{2}=d_{i j l}\left(v^{r}\right)^{2}$ if $z_{i j l}^{r}=1$, and 0 otherwise.

2. There is a speed violation on $(i, j)$ (i.e. $\left.\sum_{r \in R} z_{i j l}^{r}>u_{i j l}^{s}\right)$ :

(a) non-linear model: Due to (54)-55] $a_{i j l}^{s}=1$, hence, due to [51)-52], $S R_{i j l}^{s}=\sum_{r \in R} z_{i j l}^{r} v^{r}-$ $u_{i j l}^{s}$ for all $s \in S$. Therefore:

- $d_{i j l} \sum_{r \in R} z_{i j l}^{r} /\left(v^{r}-S R_{i j l}^{s}\right)=d_{i j l} \sum_{r \in R} z_{i j l}^{r} /\left(v^{r}-\sum_{r \in R} z_{i j l}^{r} v^{r}+u_{i j l}^{s}\right)=d_{i j l} / u_{i j l}^{s}$ if $z_{i j l}^{r}=1$, and 0 otherwise,

- $d_{i j l} \sum_{r \in R} z_{i j l}^{r}\left(v^{r}-S R_{i j l}^{s}\right)^{2}=d_{i j l} \sum_{r \in R} z_{i j l}^{r}\left(v^{r}-\sum_{r \in R} z_{i j l}^{r} v^{r}+u_{i j l}^{s}\right)^{2}=d_{i j l}\left(u_{i j l}^{s}\right)^{2}$ if $z_{i j l}^{r}=1$, and 0 otherwise.

(b) linearized model: Due to [54- 55 $a_{i j l}^{s}=1$, hence, due to 62 -63, $S F_{i j l}^{s}=1 / u_{i j l}^{s}-$ $\sum_{r \in R} z_{i j l}^{r} / v^{r}$ and due to 65-66) $S S_{i j l}^{s}=\sum_{r \in R} z_{i j l}^{r}\left(v^{r}\right)^{2}-\left(u_{i j l}^{s}\right)^{2}$ for all $s \in S$. Therefore:

- $d_{i j l} \sum_{r \in R}\left(z_{i j l}^{r} / v^{r}\right)+S F_{i j l}^{s}=d_{i j l}\left(\sum_{r \in R} z_{i j l}^{r} / v^{r}+1 / u_{i j l}^{s}-\sum_{r \in R} z_{i j l}^{r} / v^{r}\right)=d_{i j l} / u_{i j l}^{s}$ if $z_{i j l}^{r}=1$, and 0 otherwise,

- $d_{i j l} \sum_{r \in R} z_{i j l}^{r}\left(v^{r}\right)^{2}-S S_{i j l}^{s}=d_{i j l}\left(\sum_{r \in R} z_{i j l}^{r}\left(v^{r}\right)^{2}-\sum_{r \in R} z_{i j l}^{r}\left(v^{r}\right)^{2}+\left(u_{i j l}^{s}\right)^{2}\right)=d_{i j l}\left(u_{i j l}^{s}\right)^{2}$ if $z_{i j l}^{r}=1$, and 0 otherwise.

We have shown that in all possible cases, the linearized and non-linear models are equivalent. Therefore, $v_{N L P}^{*}=v_{L P}^{*}$ and the optimal solutions of the two models coincide with respect to $\left(x^{*}, z^{*}, k^{*}\right)$. 


\subsection{One-Route, Several-Speeds Strategy}

As explained in Section 3.3, the OR-SS strategy assumes that the actual maximum speeds are revealed prior to executing the routes, but after the routing decisions have been made. Here the decisions involve determining a set of routes that will remain intact regardless of the scenario, but will include a set of optimal speeds for each scenario. This problem is now modeled as a two-stage stochastic program in which the first-stage variables correspond to the routes. The variables $z_{i j l}^{r}$ that were used to choose a speed level $v^{r}$ for the segment $l$ of the arc $(i, j)$ in Section 4.2.1 will now be second-stage decision variables and redefined separately for each scenario as $z_{i j l}^{r s}$ to denote the speed level $v^{r}$. The recourse variables are potential delays experienced when serving customers. The objective is to minimize the expected cost by taking into account the scenario probabilities. The two-stage stochastic program is defined as follows, where $\mathbf{E}_{s} W(x, s)$ represents the expected cost of the objective function of second-stage problem $W(x, s)$ :

$$
\begin{aligned}
\text { Minimize } & \sum_{(i, j) \in A} \sum_{l \in L_{i j}} w f_{c} \gamma \lambda \alpha_{i j} d_{i j l} x_{i j} \\
+ & \sum_{(i, j) \in A} \sum_{l \in L_{i j}} f_{c} \gamma \lambda \alpha_{i j} d_{i j l} f_{i j} \\
+ & \sum_{s \in S} p_{s}\left(\sum_{(i, j) \in A} \sum_{l \in L_{i j}} f_{c} k \Omega V \lambda d_{i j l} \sum_{r \in R} z_{i j l}^{r s} / v^{r}\right. \\
+ & \left.\sum_{(i, j) \in A} \sum_{l \in L_{i j}} f_{c} \beta \gamma \lambda d_{i j l} \sum_{r \in R} z_{i j l}^{r s}\left(v^{r}\right)^{2}\right) \\
+ & \mathbf{E}_{s} W(x, s)
\end{aligned}
$$

subject to

$$
\begin{aligned}
& \sum_{j \in N} x_{0 j}=m \\
& \sum_{j \in N} x_{i j}=1 \quad i \in N_{0} \\
& \sum_{j \in N} x_{i j}=1 \quad j \in N_{0} \\
& \sum_{j \in N} f_{i j}-\sum_{j \in N} f_{j i}=q_{i} \quad i \in N_{0} \\
& q_{j} x_{i j} \leq f_{i j} \leq\left(Q-q_{i}\right) x_{i j} \quad(i, j) \in A
\end{aligned}
$$




$$
\begin{array}{ll}
\sum_{r \in R} z_{i j l}^{r s}=x_{i j} & (i, j) \in A, l \in L_{i j}, \quad s \in S \\
x_{i j} \in\{0,1\} & (i, j) \in A \\
f_{i j} \geq 0 & (i, j) \in A,
\end{array}
$$

where the second stage problem is defined as

$$
W(x, s)=\operatorname{minimize}\left\{\sum_{j \in N_{0}} f_{d} \delta_{j}^{s}+\sum_{j \in N} e l_{j}^{s}\right\}
$$

subject to

$$
\begin{array}{ll}
y_{i}^{s}-y_{j}^{s}+c_{i}+\sum_{r \in R} \sum_{l \in L_{i j}} d_{i j l} z_{i j l}^{r s} / v^{r} \leq M\left(1-x_{i j}\right) & i \in N, j \in N_{0}, i \neq j, \quad s \in S \\
y_{j}^{s}-c_{j}-\delta_{j}^{s}+\sum_{l \in L_{i j}} \sum_{r \in R} d_{j 0 l} z_{j 0 l}^{r s} / v^{r} \leq M\left(1-x_{j 0}\right) & j \in N_{0}, \quad s \in S \\
a_{j} \leq y_{j}^{s} \leq b_{j}+l_{j}^{s} & i \in N, j \in N_{0}, i \neq j, s \in S \\
y_{i}^{s} \geq 0 & i \in N_{0}, s \in S \\
z_{i j l}^{r s} \in\{0,1\} & (i, j) \in A, l \in L_{i j}, \quad r \in R \quad s \in S .
\end{array}
$$

The value of optimal solution yielded by the OR-SS strategy is at least as good as the optimal value of the OR-OS strategy. The relative difference between these values can be interpreted as the value of the additional information provided by the OR-SS strategy. Indeed, the gain in cost is due to the knowledge of traffic speeds before the start of the routing.

\section{Computational experiments}

The purpose of the computational experimentation carried out in this section is fourfold. First, we aim to compare the two linear models described in Section 4.2.1, which are the discrete speed and discrete recourse model, and the discrete speed and continuous recourse model. This comparison will show which of the linearization techniques is more suitable to solve the problem. Second, once the best linear stochastic model is identified, it will be compared with a deterministic model. This model considers only one scenario, where on each arc the traffic speed is the average of traffic speeds of each scenario. This comparison will highlight the advantage of using a stochastic strategy rather than a simple deterministic strategy. The relative difference between the two solutions values can 
be interpreted as the value of a stochastic solution (Birge, 1982; Birge and Louveaux, 2011). Third, in order to assess the benefit of having perfect knowledge of the traffic speeds, we will compare the OR-OS and OR-SS strategies. The difference between the solution values yielded by these two strategies can be interpreted as the value of complete information about congestion and random events. Finally, we quantify the benefits of optimizing speeds as compared with a scenario where the vehicles simply travel at the fixed traffic speed.

We have conducted experiments on the 20 benchmark instances of PRP-lib with 10 customers. All tests were performed on a computer equipped with an Intel Core i7-3770 processor, $3.4 \mathrm{GHz}$ and a RAM of 8GB. The mathematical models were solved by CPLEX 12.6.0.1 using default options. A maximum CPU time of three hours was allowed for the solution of any instance. The typical values of the parameters used in fuel emission model as described in Bektaş and Laporte (2011) are shown in Table 4, with exception of the driver wage for which we use $£ 10$ per hour as per the 2017 rate reported in (National Careers Service, 2017).

\subsection{Probability distribution and scenario generation}

As mentioned in Section 3.3, the traffic speeds are the random variables of the problem. To generate traffic speeds, we need to generate different traffic conditions on the network, which can be achieved through the use of distributions to generate vehicle speeds. Hofleitner et al. (2012) and Rakha et al. (2006) suggest that compared with other distributions, the log-normal distribution provides a good fit for vehicle speeds. This is therefore the distribution we have used to model traffic speeds on arcs.

In the computational experiments carried out, we considered three scenarios $S_{1}, S_{2}$ and $S_{3}$. Each scenario was defined using a log-normal distribution with parameters $\mu$ and $\sigma$ from which we drew traffic speeds for each arc of the graph. This distribution describes the general characteristics of the speeds for that scenario. For example, if $\mu$ is low, the traffic is low in general, although some arcs may have higher traffic speeds due to the long tail of the distribution, particularly if $\sigma$ is large. The mean and variance of a sample generated using a log-normal distribution is $\hat{\mu}=e^{\mu+\sigma^{2} / 2}$ and

455 its variance is $\hat{\sigma}^{2}=\left(e^{\sigma^{2}}-1\right) e^{2 \mu+\sigma^{2}}$. In the remainder of the paper, we will refer to the log-normal distribution by its mean $\hat{\mu}$ and variance $\hat{\sigma}^{2}$ defined above. In the first part of the computational experiments, we consider only one segment per arc (i.e. $L_{i j}=\{1\}, \forall(i, j) \in A$ ).

To generate realistic scenarios, we used the Mobile Century Data (Herrera et al., 2010) that 


\begin{tabular}{ccc}
\hline Notation & Description & Typical values \\
\hline$w$ & Curb-weight $(\mathrm{kg})$ & 6350 \\
$\varepsilon$ & Fuel-to-air mass ratio & 1 \\
$k$ & Engine friction factor $(\mathrm{kJ} / \mathrm{rev} / \mathrm{L})$ & 0.2 \\
$\Omega$ & Engine speed (rev/s) & 33 \\
$V$ & Engine displacement $(\mathrm{L})$ & 5 \\
$g$ & Gravitational constant $\left(\mathrm{m} / \mathrm{s}^{2}\right)$ & 9.81 \\
$C_{d}$ & Coefficient of aerodynamic drag & 0.7 \\
$\rho$ & Air density $\left(\mathrm{kg} / \mathrm{m}^{3}\right)$ & 1.2041 \\
$\Gamma$ & Frontal surface area $\left(\mathrm{m}^{2}\right)$ & 3.912 \\
$C_{r}$ & Coefficient of rolling resistance & 0.01 \\
$\eta_{t f}$ & Vehicle drive train efficiency & 0.4 \\
$\eta$ & Efficiency parameter for diesel engines & 0.9 \\
$f_{c}$ & Fuel and CO ${ }_{2}$ emissions cost $(£ / \mathrm{L})$ & 1.4 \\
$f_{d}$ & Driver wage $(£ / \mathrm{s})$ & 0.0028 \\
$e$ & Unit delay cost $(£ / \mathrm{s})$ & 0.0028 \\
$\kappa$ & Heating value of a typical diesel fuel $(\mathrm{kJ} / \mathrm{g})$ & 44 \\
$\Psi$ & Conversion factor $(\mathrm{g} / \mathrm{s} \mathrm{to} \mathrm{L/s)}$ & 737 \\
$\theta$ & Road angle & 0 \\
$v^{l b}$ & Speed lower bound $(\mathrm{km} / \mathrm{h})$ & 20 \\
$v^{u b}$ & Speed upper bound $(\mathrm{km} / \mathrm{h})$ & 96 \\
\hline & &
\end{tabular}

Table 4: Values of the parameters used in the emission model

provides vehicles speed collected using phones GPSs during different times. The first scenario $S_{1}$ is extracted from the data where the average speed is relatively low but has a large variance. It represents a relative congestion on some arcs while others have larger traffic speeds. The scenario $S_{1}$ is generated using a mean $\hat{\mu}=40 \mathrm{~km} / \mathrm{h}$ and a variance $\hat{\sigma}^{2}=64$. The second scenario $S_{2}$ is extracted from data where the average speed is high but values over the arcs have high fluctuation. It is generated with $\hat{\mu}=70$ and $\hat{\sigma}^{2}=144$. The last scenario is generated using $\hat{\mu}=55$ and $\hat{\sigma}^{2}=100$ 
The traffic speed generated $u_{i j}^{s}$ for all $\operatorname{arcs}(i, j) \in A$ and all scenarios $s \in S$ must lie within the interval $20 \leq u_{i j}^{s} \leq 96$ required by law for delivery vehicles. Below is an example of a complete graph defined with a single depot and three customers, where on each arc the traffic speed are drawn from scenario $S_{2}$ :

$$
\left[\begin{array}{cccc}
0 & 87 & 20 & 45 \\
58 & 0 & 26 & 51 \\
29 & 59 & 0 & 29 \\
58 & 24 & 61 & 0
\end{array}\right] .
$$

For all comparisons provided in this section, every instance was solved on three different, and randomly generated realizations of $S_{1}, S_{2}$ and $S_{3}$, the results displayed on the tables represents the average of all these tests. This methodology allow the results to be meaningful and do not rely on a particular realization of the scenarios. It also enables the assessment of the robustness of the solution algorithms with respect to changes in traffic conditions.

\subsection{Comparative results for the discretized and continuous recourse strategies}

We now present the results of experiments in which each of the 20 instances was solved once by the discretized recourse model, and once by the continuous recourse model, both described in Section 4.2.1. The results are presented in Table 5 which displays the optimal values for the instances (in $£)$ and solution times in seconds to optimality. The gap (\%) is the percentage of the continuous recourse optimal solution value saved with respect to the optimal solution value of the discrete recourse model. We can see from Table 5 that the fixed continuous recourse model outperforms the discretized recourse model by an average of $4.95 \%$ in terms of optimal solution value. This table also shows that the continuous recourse model is more than twice faster on average than the discretized recourse model. On instance UK10_15, the discretized recourse model could not yield a proven optimal solution within the time limit of three hours; in this case, we report the best known solution value. Given this result, OR-OS, will henceforth refer to the discretized speed and continuous recourse model.

\subsection{Comparative results for the OR-OS strategy with the deterministic model and OR-SS strategy}

Here, we present the results of experiments in which each of the 20 instances is solved by the deterministic and the OR-SS approaches, both described in Section 4.2.1 and 4.3. The relative difference in cost between the OR-OS and deterministic approaches is the Value of the Stochastic 


\begin{tabular}{c|c|c|c|c|c}
\hline Instance & \multicolumn{2}{|c|}{ Discrete recourse } & \multicolumn{2}{c|}{ Continuous recourse } & \\
\hline & Optimal value & Seconds & Optimal value & Seconds & Gap (\%) \\
\hline UK10_01 & 261.6 & 4799.0 & 251.3 & 1119.7 & 4.1 \\
UK10_02 & 327.6 & 2505.3 & 308.4 & 1844.7 & 6.2 \\
UK10_03 & 295.6 & 2682.0 & 282.3 & 818.7 & 4.7 \\
UK10_04 & 284.4 & 1736.3 & 270.3 & 711.0 & 5.2 \\
UK10_05 & 240.4 & 3061.0 & 232.1 & 719.0 & 3.6 \\
UK10_06 & 343.4 & 4352.0 & 328.9 & 2092.3 & 4.4 \\
UK10_07 & 288.5 & 2250.3 & 277.7 & 1004.7 & 3.9 \\
UK10_08 & 330.0 & 1607.5 & 313.2 & 487.0 & 5.4 \\
UK10_09 & 259.6 & 763.7 & 246.4 & 213.3 & 5.4 \\
UK10_10 & 293.1 & 2319.7 & 278.0 & 587.3 & 5.4 \\
UK10_11 & 439.0 & 1206.3 & 409.8 & 1083.3 & 7.1 \\
UK10_12 & 260.2 & 849.7 & 248.4 & 829.3 & 4.8 \\
UK10_13 & 289.5 & 1408.3 & 279.7 & 1431.7 & 3.5 \\
UK10_14 & 282.3 & 5305.7 & 267.0 & 2963.0 & 5.7 \\
UK10_15 & 210.7 & 10800.0 & 199.5 & 3381.0 & 5.6 \\
UK10_16 & 252.0 & 4616.0 & 243.8 & 1447.0 & 3.4 \\
UK10_17 & 255.6 & 1235.3 & 246.5 & 347.7 & 3.7 \\
UK10_18 & 242.5 & 3574.0 & 225.8 & 1062.0 & 7.4 \\
UK10_19 & 266.3 & 2974.3 & 251.6 & 573.3 & 5.8 \\
UK10_20 & 244.1 & 4259.0 & 235.8 & 1373.0 & 3.6 \\
\hline Average & 283.32 & 3115.28 & 269.82 & 1204.45 & 4.95 \\
\hline & & & & & \\
\hline
\end{tabular}

Table 5: Comparison between the solutions of the discretized and continuous recourse models

Solution (VSS) (Birge, 1982). It represents the percentage cost saved when solving a stochastic model rather than a deterministic one. The VSS is computed as follows:

$$
V S S=\frac{\text { Deterministic solution cost }- \text { Stochastic solution cost }}{\text { Stochastic solution cost }} \times 100 .
$$

In Table 6, we show the optimal solutions values (in £), the solution times in seconds, and 
the VSS. We see that the stochastic solutions are on average better than the deterministic ones by $7.48 \%$. The largest saving $17.7 \%$, is obtained by the OR-OS strategy is achieved on instance UK10_15. The average solution time to optimality of the deterministic model is 47.03 seconds, while the same statistic for the OR-OS model is 1204.45 seconds.

The OR-SS strategy reduces the total cost obtained by the OR-OS on average by $1.27 \%$. This improvement is the saving achieved when perfect information about the traffic speeds is available, but this information is in practice very difficult to acquire. However, the results of these tests show that the use OR-OS strategy can provide solutions that are on average less than two percent more expensive than those based on perfect information. Comparing the times required to solve the instances to optimality, we see that OR-SS strategy requires 191.05 seconds on average, while the OR-OS strategy is slower, with an average solution time of 1204.45 seconds. This difference is justified by the fact that no speed recourse decisions are needed under the OR-SS strategy, and hence fewer solutions need to be checked to prove the optimality of the current solution.

\subsection{Correlation between total cost and fuel cost savings}

To gain more insight, we provide a pairwise comparison of six scenarios, two with low means equal to $30 \mathrm{~km} / \mathrm{h}$ and $40 \mathrm{~km} / \mathrm{h}$, two with higher means equal to $55 \mathrm{~km} / \mathrm{h}$ and $70 \mathrm{~km} / \mathrm{h}$, and with variances equal to either 64 or 144, yielding eight combinations. The tests show how the traffic conditions affect both the fuel consumption and the total cost of the solutions. The tests are run on the instance UK10_01 and the results are reported in Table 7 where each entry corresponds to a pair of scenarios, and contains two rows. The first row reports the percentage reduction in fuel consumption achieved by the OR-OS strategy over the deterministic approach, and it is displayed in the form (minimum, average, maximum). A negative value indicates that the deterministic approach performed better than the OR-OS strategy. The second row reports similar statistics for the total solution cost.

The results shown in Table 7 suggest that the savings in total cost and in fuel consumption are not necessarily correlated. In fact, a large reduction in total cost does not imply a large reduction in fuel consumption. In this respect, Demir et al. (2014a) pointed out that the objective function is composed of two cost components: fuel consumption and driver cost. These are often conflicting, and the latter plays a more important role in achieving reductions in the overall cost. The results indicate that the largest savings both in fuel and in driver cost are achieved when the average speed 


\begin{tabular}{|c|c|c|c|c|c|c|}
\hline \multirow[t]{2}{*}{ Instance } & \multicolumn{3}{|c|}{ Deterministic } & \multicolumn{3}{|c|}{ OR-SS } \\
\hline & $\begin{array}{l}\text { Optimal } \\
\text { value }\end{array}$ & Seconds & VSS (\%) & $\begin{array}{l}\text { Optimal } \\
\text { value }\end{array}$ & Seconds & Gap (\%) \\
\hline UK10_01 & 265.4 & 33.0 & 5.6 & 246.3 & 154.3 & -2.0 \\
\hline UK10_02 & 332.6 & 37.7 & 7.8 & 303.5 & 138.0 & -1.6 \\
\hline UK10_03 & 297.3 & 64.3 & 5.3 & 278.8 & 158.3 & -1.3 \\
\hline UK10_04 & 288.7 & 43.3 & 6.8 & 267.4 & 132.3 & -1.1 \\
\hline UK10_05 & 241.5 & 52.5 & 4.1 & 230.4 & 187.0 & -0.7 \\
\hline UK10_06 & 352.3 & 78.7 & 7.1 & 325.2 & 358.7 & -1.1 \\
\hline UK10_07 & 295.5 & 35.3 & 6.4 & 274.5 & 144.3 & -1.2 \\
\hline UK10_08 & 332.4 & 16.0 & 6.1 & 309.5 & 57.0 & -1.2 \\
\hline UK10_09 & 256.5 & 10.7 & 4.1 & 243.3 & 30.0 & -1.3 \\
\hline UK10_10 & 294.0 & 18.0 & 5.8 & 273.5 & 84.7 & -1.6 \\
\hline UK10_11 & 444.6 & 41.7 & 8.5 & 406.3 & 218.0 & -0.9 \\
\hline UK10_12 & 263.5 & 45.3 & 6.1 & 245.9 & 185.3 & -1.0 \\
\hline UK10_13 & 290.6 & 71.3 & 3.9 & 275.0 & 333.0 & -1.7 \\
\hline UK10_14 & 282.1 & 105.3 & 5.7 & 263.3 & 427.7 & -1.4 \\
\hline UK10_15 & 234.9 & 58.7 & 17.7 & 196.0 & 498.0 & -1.8 \\
\hline UK10_16 & 254.9 & 49.0 & 4.5 & 242.0 & 113.3 & -0.7 \\
\hline UK10_17 & 281.4 & 13.7 & 14.2 & 244.1 & 58.0 & -1.0 \\
\hline UK10_18 & 259.3 & 53.7 & 14.8 & 223.1 & 143.7 & -1.2 \\
\hline UK10_19 & 277.7 & 30.0 & 10.4 & 248.4 & 101.0 & -1.3 \\
\hline UK10_20 & 246.7 & 82.3 & 4.6 & 232.5 & 298.3 & -1.4 \\
\hline Average & 289.59 & 47.03 & 7.48 & 266.45 & 191.05 & -1.27 \\
\hline
\end{tabular}

Table 6: Performance of deterministic and OR-SS approaches and comparison with OR-OS

across all scenarios is less than the the globally optimal speed $v^{*}$ that minimizes fuel consumption (which depends on the type of vehicle and is between 50 and $60 \mathrm{~km} / \mathrm{h}$ in our case), otherwise the savings in fuel consumption are less than $0.6 \%$ on average. The results also show that the deterministic approach was able to save fuel on some instances, but this saving is achieved at the 


\begin{tabular}{lcc}
\hline & $\hat{\mu}=40, \hat{\sigma}^{2}=144$ & $\hat{\mu}=70, \hat{\sigma}^{2}=144$ \\
\hline$\hat{\mu}=30, \hat{\sigma}^{2}=64$ & $(-0.2 \%, 0.4 \%, 1.2 \%)$ & $(-1.8 \%, 0.1 \%, 2.2 \%)$ \\
\cline { 2 - 3 } & $(9.3 \%, 12.9 \%, 15.6 \%)$ & $(7.6 \%, 15.8 \%, 20.8 \%)$ \\
\hline$\hat{\mu}=30, \hat{\sigma}^{2}=144$ & $(-1.9 \%,-1.3 \%,-0.8 \%)$ & $(-1.2 \%,-0.1 \%, 1.3 \%)$ \\
\hline$\hat{\mu}=55, \hat{\sigma}^{2}=64$ & $(3.2 \%, 7.6 \%, 13.3 \%)$ & $(6.7 \%, 12.7 \%, 16.2 \%)$ \\
\cline { 2 - 3 } & $(3.9 \%, 5.7 \%, 9.3 \%)$ & $(10.1 \%, 11.8 \%, 13.2 \%)$ \\
\hline$\hat{\mu}=55, \hat{\sigma}^{2}=144$ & $(-0.5 \%, 2.1 \%, 5.6 \%)$ & $(-1.7 \%, 0.0 \%, 2.7 \%)$ \\
\cline { 2 - 3 } & $(4.4 \%, 11.4 \%, 20.1 \%)$ & $(7.2 \%, 13.5 \%, 24.7 \%)$ \\
\hline
\end{tabular}

Table 7: Comparison between deterministic and OR-OS solution values

expense of the overall cost. Finally, when the average speed of one scenario is lower than $v^{*}$ and the other is higher than $v^{*}$, the two approaches yield similar fuel consumptions, but the OR-OS strategy performs better in terms of the overall cost.

\subsection{The value of optimizing speed}

Most papers relevant to the stochastic VRP, such as those reviewed in Section 2.2, do not consider speed as a decision variable. This means that if arc $(i, j)$ is chosen in the first stage, then it is assumed that the vehicle will travel on that arc at a traffic speed that is scenario dependent. From a decision-making point of view, this does not allow any flexibility in changing vehicle speeds and does not take into account the flexibility of reducing speed to save on fuel cost. From a methodological point of view, this is a special case of our approach where the speed decision variables are fixed to the traffic speeds on all arcs. 
To quantify the value of optimizing speed, we present results using the first 10 instances of the $\left(\hat{\mu}=40, \hat{\sigma}^{2}=64\right),\left(\hat{\mu}=30, \hat{\sigma}^{2}=64\right)$.

\begin{tabular}{c|ccc|ccc|ccc}
\hline Setting & \multicolumn{3}{|c|}{ Minimum (\%) } & \multicolumn{3}{|c}{ Average (\%) } & \multicolumn{3}{c}{ Maximum (\%) } \\
& Min & Av & Max & Min & Av & Max & Min & Av & Max \\
\hline 1 & 0.0 & 0.0 & 0.0 & 0.0 & 0.1 & 0.3 & 0.0 & 0.3 & 0.4 \\
2 & 0.0 & 0.1 & 0.3 & 0.1 & 0.3 & 0.8 & 0.2 & 0.7 & 1.5 \\
3 & 0.0 & 0.0 & 0.0 & 0.1 & 0.1 & 0.3 & 0.1 & 0.3 & 0.5 \\
4 & 0.0 & 0.0 & 0.2 & 0.1 & 0.2 & 0.4 & 0.1 & 0.4 & 0.7 \\
5 & 0.0 & 0.0 & 0.0 & 0.0 & 0.1 & 0.2 & 0.1 & 0.2 & 0.4 \\
\hline
\end{tabular}

Table 8: Savings achieved by considering speed as a decision variable by optimized speeds against the case where speeds are fixed to the traffic speed value prescribed by the scenarios. Table 8 shows the results over 3 realizations of each scenario in terms of the minimum, average and maximum savings, presented under the three main columns. For each of the columns, we again provide the minimum, average and maximum savings obtained. For example, the column titled Minimum and sub-column titled Av shows the average minimum saving achieved across the 10 instances and the 3 realizations of the scenarios. The values are expressed in percentage of the objective value which includes only fuel costs. Each row represents a setting, numbered 1-5, that are generated using the scenarios where settings 1 and 2 include one, settings 3,4 and 5 include two scenarios. The scenarios are defined by the following distributions $\left(\hat{\mu}=40, \hat{\sigma}^{2}=64\right)$; $\left(\hat{\mu}=70, \hat{\sigma}^{2}=144\right) ;\left(\hat{\mu}=70, \hat{\sigma}^{2}=144\right),\left(\hat{\mu}=30, \hat{\sigma}^{2}=64\right) ;\left(\hat{\mu}=70, \hat{\sigma}^{2}=144\right),\left(\hat{\mu}=80, \hat{\sigma}^{2}=164\right) ;$

Table 8 shows that considering speed as a decision variable can result in average savings of up to $0.8 \%$ in terms of the total cost. Low savings are due to the fact that driver cost is the largest cost in the objective function (Bektaş and Laporte, 2011). With the introduction of ATs, the driver is not in control of the vehicle on the highways, and hence driver cost can be removed from the objective function to minimize exclusively fuel consumption and GHG emissions.

In Table 9, we repeat the experiment, with the same scenarios described above. This time however, we do not consider driver wage in the objective function. We can see that, on average, considering speed as a decision variable can achieve savings on fuel consumption and GHG emissions 
between $0.7 \%$ and $3.8 \%$. The average minimum saving is achieved in setting 1 , whereas the average maximum is $4.4 \%$ is made on setting 2 , where the maximum saving achieved is $8.5 \%$. This shows that the savings made are highly correlated with general trend of the traffic speed on the network. Indeed, when the traffic speed is high, the fuel consumption is also high. Therefore, if the vehicle has to travel on all arcs at the traffic speed, it will not choose some arcs that have high speed, which in turn will increase the fuel consumption. This situation is avoided by considering speed as a decision variable, since the vehicle then has the ability to travel at speed that is lower than the traffic speed.

\begin{tabular}{c|ccc|ccc|ccc}
\hline \multirow{2}{*}{ Setting } & \multicolumn{3}{|c|}{ Minimum (\%) } & \multicolumn{3}{c|}{ Average (\%) } & \multicolumn{3}{c}{ Maximum (\%) } \\
& Min & Av & Max & Min & Av & Max & Min & Av & Max \\
\hline 1 & 0.0 & 0.6 & 1.4 & 0.7 & 1.5 & 3.8 & 1.1 & 2.5 & 7.9 \\
2 & 0.0 & 1.3 & 2.1 & 1.7 & 2.7 & 3.6 & 2.5 & 4.4 & 8.4 \\
3 & 0.5 & 1.1 & 1.6 & 1.2 & 1.8 & 2.5 & 1.7 & 2.6 & 4.2 \\
4 & 0.5 & 1.3 & 2.6 & 1.5 & 2.2 & 3.3 & 1.9 & 3.2 & 4.5 \\
5 & 0.2 & 0.8 & 1.8 & 0.8 & 1.4 & 1.9 & 1.5 & 2.0 & 2.4 \\
\hline
\end{tabular}

Table 9: Savings achieved by considering speed as a decision variable without driver

\subsection{The effect of variability in traffic speed}

This section reports results when more than one segment is assumed for each arc in the network to be able to evaluate the effect of any speed variability.

We consider a road network where we generate a traffic speed for two segments per arc and then solve the problem using the OR-OS approach. To compare this approach, we consider another network where the traffic speed on one arc is the average of its two segments and solve using the same approach. From this test we see the difference in cost of the two approaches which represents the accuracy in handling speed variability brought by considering more segments.

Table 10 shows the results of these two settings. We see that, on average, considering two segments increased the accuracy by $8 \%$ compared to only one segment per arc. We also notice that ignoring speed variability may result in an over-optimistic approach that underestimates the real cost. From a computational complexity point of view, considering two segments slows down the solution time by a factor of almost six. 


\begin{tabular}{c|c|c|c|c|c}
\hline Instance & \multicolumn{2}{|c|}{ Two segments } & \multicolumn{2}{c|}{ One segment } & \\
\hline & Optimal value & Seconds & Optimal value & Seconds & Gap (\%) \\
\hline UK10_01 & 247.3 & 7500.0 & 230.3 & 804.0 & 7.4 \\
UK10_02 & 309.3 & 8634.0 & 284.4 & 797.0 & 8.8 \\
UK10_03 & 285.4 & 6475.0 & 268.2 & 1008.0 & 6.4 \\
UK10_04 & 275.4 & 6385.0 & 253.9 & 826.0 & 8.5 \\
UK10_05 & 252.1 & 8690.0 & 237.2 & 3852.0 & 6.3 \\
UK10_06 & 322.5 & 7916.5 & 292.1 & 1347.0 & 10.4 \\
UK10_07 & 278.2 & 3826.0 & 254.8 & 410.5 & 9.2 \\
UK10_08 & 325.6 & 1813.0 & 299.1 & 223.3 & 8.9 \\
UK10_09 & 250.3 & 3283.0 & 232.0 & 239.7 & 7.9 \\
UK10_10 & 272.6 & 2659.0 & 254.7 & 274.3 & 7.0 \\
\hline Average & 281.91 & 5718.15 & 260.67 & 978.18 & 8.0 \\
\hline
\end{tabular}

Table 10: Comparison between network with two segments per arc and with one segment per arc The continuous recourse strategy was also compared with its deterministic counterpart in which traffic speeds are the averages across all scenarios. This enabled us to estimate the value of solving a 
stochastic model as opposed to a deterministic one. The continuous recourse model yielded average cost savings of $7.48 \%$.

We have also studied a decision making process in which the routing decisions are made before the information about stochastic events becomes available, but speeds can be chosen after the realizations of the scenarios. While this scenario is less likely to occur in practice, it was implemented to evaluate the value of perfect information on traffic condition. When this model was solved on the same instances, only $1.27 \%$ of the total cost was saved with respect to the continuous recourse model on average. Finally, we have proposed a way to represent traffic speeds variability by considering several segments on a given arc. We showed that considering two segments per arc can increase the accuracy of the solution by $8.0 \%$ on average.

\section{Acknowledgments}

The authors gratefully acknowledge funding provided by the Southampton Business School and by the Canadian Natural Sciences and Engineering Research Council under grants 2015-06189 and 436014-2013. Thanks are due to the referees for their valuable comments.

\section{References}

de Almeida Correia, G.H., van Arem, B., 2016. Solving the user optimum privately owned automated vehicles assignment problem (UO-POAVAP): A model to explore the impacts of self-driving vehicles on urban mobility. Transportation Research Part B: Methodological 87, 64-88.

Barth, M., Boriboonsomsin, K., 2008. Real-world carbon dioxide impacts of traffic congestion. Transportation Research Record: Journal of the Transportation Research Board, 163-171.

Bektaş, T., Laporte, G., 2011. The pollution-routing problem. Transportation Research Part B:

Methodological 45, 1232-1250.

Bektaş, T., Demir, E., Laporte, G., 2016. Green vehicle routing, in: Psaraftis, H.N. (Ed.), Green Transportation Logistics: The Quest for Win-Win Solutions. Springer, Heidelberg, pp. 243-265.

Birge, J.R., 1982. The value of the stochastic solution in stochastic linear programs with fixed recourse. Mathematical Programming 24, 314-325. 
Birge, J.R., Louveaux, F.V., 2011. Introduction to Stochastic Programming. Springer Science \& Business Media, Heidelberg.

Cordeau, J.F., Laporte, G., Savelsbergh, M.W., Vigo, D., 2007. Vehicle routing, in: Barnhart, C., Laporte, G. (Eds.), Handbooks in Operations Research and Management Science : Transportation. North-Holland, Amsterdam. volume 14, pp. 367-428.

Costello, B., Suarez, R., 2015. Truck driver shortage analysis 2015. Technical Report. The American Trucking Associations. Arlington, VA.

Dabia, S., Demir, E., Van Woensel, T., 2016. An exact approach for a variant of the pollution-routing problem. Transportation Science 51, 607-628.

Demir, E., Bektaş, T., Laporte, G., 2012. An adaptive large neighborhood search heuristic for the pollution-routing problem. European Journal of Operational Research 223, 346-359.

Demir, E., Bektaş, T., Laporte, G., 2014a. The bi-objective pollution-routing problem. European Journal of Operational Research 232, 464-478.

Demir, E., Bektaş, T., Laporte, G., 2014b. A review of recent research on green road freight transportation. European Journal of Operational Research 237, 775-793.

Dijkstra, E.W., 1959. A note on two problems in connexion with graphs. Numerische Mathematik 1, 269-271.

Eglese, R.W., Bektaş, T., 2014. Green vehicle routing, in: Toth, P., Vigo, D. (Eds.), Vehicle Routing: Problems, Methods, and Applications. MOS-SIAM Series on Optimization, Philadelphia. volume 18 , pp. $437-458$.

Ehmke, J.F., Campbell, A.M., Thomas, B.W., 2016. Data-driven approaches for emissions-minimized paths in urban areas. Computers \& Operations Research 67, 34-47.

Eshtehadi, R., Fathian, M., Demir, E., 2017. Robust solutions to the pollution-routing problem with demand and travel time uncertainty. Transportation Research Part D: Transport and Environment $51,351-363$. 
Fagnant, D.J., Kockelman, K.M., 2014. The travel and environmental implications of shared autonomous vehicles, using agent-based model scenarios. Transportation Research Part C: Emerging Technologies 40, 1-13.

Fang, Y., Chu, F., Mammar, S., Che, A., 2013. An optimal algorithm for automated truck freight transportation via lane reservation strategy. Transportation Research Part C: Emerging Technologies 26, 170-183.

Flämig, H., 2016. Autonomous vehicles and autonomous driving in freight transport, in: Markus Maurer, Joseph Christian Gerdes, B.L.H.W. (Ed.), Autonomous Driving. Springer, Heidelberg, pp. $365-385$.

Franceschetti, A., Honhon, D., Van Woensel, T., Bektaş, T., Laporte, G., 2013. The time-dependent pollution-routing problem. Transportation Research Part B: Methodological 56, 265-293.

Fukasawa, R., He, Q., Song, Y., 2016. A disjunctive convex programming approach to the pollutionrouting problem. Transportation Research Part B: Methodological 94, 61-79.

Gendreau, M., Jabali, O., Rei, W., 2016. Future research directions in stochastic vehicle routing - 50th anniversary invited article. Transportation Science 50, 1163-1173.

Herrera, J.C., Work, D.B., Herring, R., Ban, X.J., Jacobson, Q., Bayen, A.M., 2010. Evaluation of traffic data obtained via gps-enabled mobile phones: The mobile century field experiment. Transportation Research Part C: Emerging Technologies 18, 568-583.

Hofleitner, A., Herring, R., Bayen, A., 2012. Probability distributions of travel times on arterial networks: a traffic flow and horizontal queuing theory approach, in: 91st Transportation Research Board Annual Meeting, Washington DC.

Huang, Y., Zhao, L., Van Woensel, T., Gross, J.P., 2017. Time-dependent vehicle routing problem with path flexibility. Transportation Research Part B: Methodological 95, 169-195.

Hwang, T., Ouyang, Y., 2015. Urban freight truck routing under stochastic congestion and emission considerations. Sustainability 7, 6610-6625.

Kramer, R., Subramanian, A., Vidal, T., Lucídio dos Anjos, F.C., 2015. A matheuristic approach for the pollution-routing problem. European Journal of Operational Research 243, 523-539. 
Laporte, G., Louveaux, F.V., Mercure, H., 1992. The vehicle routing problem with stochastic travel times. Transportation Science 26, 161-170.

Lin, C., Choy, K.L., Ho, G.T., Chung, S., Lam, H., 2014. Survey of green vehicle routing problem: Past and future trends. Expert Systems with Applications 41, 1118-1138.

Maden, W., Eglese, R.W., Black, D., 2010. Vehicle routing and scheduling with time-varying data: A case study. Journal of the Operational Research Society 61, 515-522.

National Careers Service, 2017. Large goods vehicle driver. https://nationalcareersservice. direct.gov.uk/job-profiles/large-goods-vehicle-driver. Accessed on 30.11.2017.

Norstad, I., Fagerholt, K., Laporte, G., 2011. Tramp ship routing and scheduling with speed optimization. Transportation Research Part C: Emerging Technologies 19, 853-865.

Prescott-Gagnon, É., Desaulniers, G., Rousseau, L.M., 2009. A branch-and-price-based large neighborhood search algorithm for the vehicle routing problem with time windows. Networks 54 , 190-204.

Psaraftis, H.N., 2016. Green Transportation Logistics: The Quest for Win-win Solutions. Springer, New York.

Qian, J., Eglese, R.W., 2014. Finding least fuel emission paths in a network with time-varying speeds. Networks 63, 96-106.

Qian, J., Eglese, R.W., 2016. Fuel emissions optimization in vehicle routing problems with timevarying speeds. European Journal of Operational Research 248, 840-848.

Rakha, H.A., El-Shawarby, I., Arafeh, M., Dion, F., 2006. Estimating path travel-time reliability, in: IEE Intelligent Transportation Systems Conference, pp. 236-241.

Scora, G., Barth, M., 2006. Comprehensive modal emissions model (CMEM), version 3.01. User's guide. Technical Report. Centre for Environmental Research and Technology. University of California, Riverside.

Singh, S., 2015. Critical reasons for crashes investigated in the national motor vehicle crash causation survey. Technical Report DOT HS 812 115. National Highway Traffic Safety Administration. Washington, DC. 
Subramanian, A., Drummond, L.M.d.A., Bentes, C., Ochi, L.S., Farias, R., 2010. A parallel heuristic for the vehicle routing problem with simultaneous pickup and delivery. Computers \& Operations Research 37, 1899-1911.

Taş, D., Dellaert, N., Van Woensel, T., De Kok, T., 2013. Vehicle routing problem with stochastic travel times including soft time windows and service costs. Computers \& Operations Research 40, $214-224$

The Guardian, 2016. Convoy of self-driving trucks completes first european cross-border trip https://www.theguardian.com/technology/2016/apr/07/ convoy-self-driving-trucks-completes-first-european-cross-border-trip. Accessed on 30.11.2017.

Tsugawa, S., 2012. Energy its: What we learned and what we should learn. on705 linepubs. trb. org. http://onlinepubs.trb.org/onlinepubs/conferences/2012/Automation/ presentations/Tsugawa.pdf. Accessed on 30.11.2017.

Vanholme, B., Gruyer, D., Lusetti, B., Glaser, S., Mammar, S., 2013. Highly automated driving on highways based on legal safety. IEEE Transactions on Intelligent Transportation Systems 14, $333-347$.

710 Wired.com, 2016. Uber's self-driving truck makes its first delivery: 50.000 beers. https://www . wired com/2016/10/ubers-self-driving-truck-makes-first-delivery-50000-beers/. Accessed on 30.11.2017.

Wu, P., Chu, F., Che, A., Fang, Y., 2017. An efficient two-phase exact algorithm for the automated truck freight transportation problem. Computers \& Industrial Engineering 110, 59-66. 